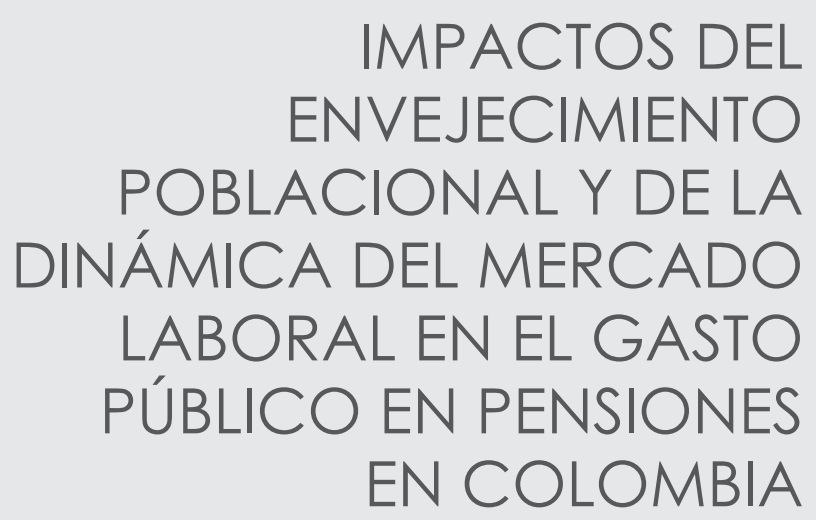

Andrés Pérez-Vasquez Universidad Santo Tomás Colombia

Juan Sandoval-Castaño Universidad Nacional de Colombia Colombia 
Panorama Económico, Vol. 25 - No. 4 (Octubre - Diciembre de 2017), pp. 493-518

Andrés Pérez-Vasquez

Juan Sandoval-Castaño

\title{
Impactos del envejecimiento poblacional y de la dinámica del mercado laboral en el
} gasto público en pensiones en Colombia

\section{Resumen}

Este documento tiene como objetivo estimar los impactos del envejecimiento poblacional y de la dinámica del mercado laboral en el gasto público en pensiones en el corto y mediano plazo mediante la simulación de diferentes escenarios de formalidad y productividad laboral teniendo en cuenta los factores institucionales del sistema pensional colombiano. Se concluye que no basta con el aumento en la formalización laboral para reducir el gasto público en pensiones, ya que en el mediano plazo es la productividad laboral la variable esencial en la sostenibilidad financiera del sistema, si y solamente si sus aumentos se acompañan con un incremento en los niveles de salario y cotización.

Palabras clave: Pensiones, Envejecimiento Poblacional, Formalización Laboral, Productividad Laboral, Gasto en Pensiones

Clasificación JEL: H55, J14, E62, H75

\section{Aging impacts and labor market dynamics on pension public expenditures in Colombia}

\begin{abstract}
This document aims to estimate the impacts of population aging and labor market dynamics on public spending on pensions in the short and medium term by simulating different formality and labor productivity scenarios taking into account the institutional factors of the pension system Colombian. It is concluded that the increase in labor formalization is not enough to reduce public expenditure on pensions, since in the medium term labor productivity is the essential variable in the financial sustainability of the system, if and only if its increases are accompanied by a increase in salary and contribution levels.

Keywords: Pensions, Population Aging, Labor Formalization, Labor Productivity

JEL Classification: H55, J14, E62, H75
\end{abstract}

\section{Impacts du vieillissement et dynamique du marché du travail sur les dépenses publiques des retraites en Colombie}

\section{Résumé}

Ce document vise à estimer les impacts du vieillissement de la population et de la dynamique du marché du travail sur les dépenses publiques de pension à court et moyen terme en simulant différents scénarios de formalité et de productivité du travail tenant compte des facteurs institutionnels du système de retraite Colombien. Il est conclu que l'augmentation de la formalisation de la maind'œuvre n'est pas suffisante pour réduire les dépenses publiques consacrées aux retraites, car la productivité du travail est la variable essentielle de la viabilité financière du système à moyen terme augmentation des salaires et des cotisations.

Mots-clés: Pensions, vieillissement de la population, formalisation du travail, productivité du travail Nomenclature JEL: H55, J14, E62, H75 


\section{Impactos del envejecimiento poblacional y de la dinámica del mercado laboral en el gasto público en pensiones en Colombia ${ }^{1}$}

INFORMACIÓN DEL ARTÍCULO

Recepción de artículo: 09/04/2017

Concepto de evaluación: 15/05/2017

Aceptación de artículo: 26/06/2017
Andrés Pérez-Vasquez*

Universidad Santo Tomás

Colombia

Juan Sandoval-Castaño

Universidad Nacional de Colombia

Colombia

\section{INTRODUCCIÓN}

En términos institucionales las reformas al sistema de pensiones colombiano (Ley 100 de 1993, Leyes 793 y 863 de 2003 y el Acto Legislativo de 2005), no han sido efectivas, en los dos objetivos propuestos: 1) incrementar la cobertura pensional y 2) contrarrestar el desequilibrio financiero derivado de aumentos en la cobertura de la afiliación ${ }^{2}$. El sistema de pensiones colombiano tiene problemas estructurales, de cobertura, equidad, suficiencia, y resolver estos problemas implica desafíos en términos de la sostenibilidad financiera del mismo ${ }^{3}$.

Es importante subrayar que el proceso de transición demográfica marcará un cambio sustancial en la estructura poblacional del país. Según Naciones Unidas (2012), en Colombia, por cada joven habrá en promedio 37 adultos mayores causando un aumento de la demanda de servicios de seguridad social en salud, pensiones y cuidado. A sí mismo, el envejecimiento poblacional implicará en el mediano y largo plazo una reducción en la fuerza laboral colombiana ocasionando un aumento en el índice de dependencia

\footnotetext{
* Autor para correspondencia

Correos electrónicos: vpma90@gmail.com*,jpsandovalc@unal.edu.co

1 Esta investigación se realizó en el Grupo de Investigación en Protección Social y Salud Pública, como beneficiaria de la BecaPasantía del programa de Jóvenes Investigadores e Innovadores, enmarcada en la Convocatoria de Colciencias No 566 de 2012. Igualmente, es menester de los autores mencionar la valiosa colaboración y asesoría del centro de consultoría de la Facultad de Estadística a cargo del profesor Andrés Felipe Ortiz, y su equipo conformado por los estadísticos en formación Paola Andrea Pérez, Juan Sebastián Garcés \& Wilson Colmenares, a ellos un profundo agradecimiento y admiración en su labor.

2 Para mayor profundización en el tema véase Schmidt (1997), Helmsdorff (2007), Schutt (2011), Moreno y Ortiz (2010), Bustamante (2006), Sánchez (2008), Uribe (2002) Salazar (2007), Echeverry et al (2001).

3 Adicionalmente, las excepciones de ley permitieron al sistema dar beneficios a grupos de trabajadores especiales por su condición de riesgo, ya sea profesional o administrativo, en pro a guardar el precepto de equidad. Sin embargo, la accesibilidad de las cifras en pensiones para regímenes especiales es bastante compleja, por lo cual no se incluye en esta investigación.
} 
económica. Como lo proyecta Naciones Unidas (2012) para Colombia por cada persona que esté trabajando o buscando trabajo habrá en promedio 76 adultos mayores para finales de siglo XXI, lo que crea presiones en el mercado laboral ligadas a la necesidad de incrementar la productividad.

El principal problema del sistema pensional colombiano son las características de su mercado laboral. Según las cifras del DANE 4 , el 49,36\% de los ocupados se encuentra en la informalidad lo cual significa una menor productividad laboral, débiles remuneraciones, baja calificación de la mano de obra, multiplicidad de riesgos y una grave desprotección de la fuerza laboral en términos de seguridad social en salud, pensiones y riesgos profesionales. Mejorar la cobertura pensional no pasa entonces solamente por incrementar la formalidad en el trabajo para mejorar la dinámica laboral y asegurar la protección social puesto que la baja productividad no permite aumentar los niveles de salarios y por lo tanto los índices de cotización. Esto implica que mayores niveles de cobertura en pensiones requieren procesos estructurales de modernización de la estructura productiva colombiana.

Con base en lo anterior, la presente investigación tiene como objetivo identificar los retos de estimar el incremento en el gasto público en pensiones en el mediano plazo en distintos escenarios de envejecimiento poblacional, formalización y productividad del trabajo y reformas institucionales del sistema de pensiones (diferentes grados de cobertura).

En este sentido, la investigación contempla la simulación de cuatro escenarios, uno base y tres alternativos. En el primero de ellos, el escenario base, se predice un incremento

4 Cifras a Diciembre de 2013. del gasto en pensiones en un 7\% del PIB a 2030 teniendo en cuenta proyecciones de las variables con base en su tendencia. Por otro lado, en un segundo escenario suponemos incrementos de la formalidad laboral del 1\%,2\% y del $5 \%$ anual que tendrá un impacto similar al escenario base en el gasto en pensiones situándose en un 7\% del PIB a 2030. Por otra parte en un tercer escenario suponemos un incremento de la productividad del $1 \%$, $2 \%$ y $5 \%$ anual que repercutirá en una disminución del gasto público en pensiones de un 6,5\%, 5\% y 7\% del PIB, respectivamente. Por último, en el cuarto ejercicio se simula un escenario asistencialista donde se considera que el Estado subsidia una pensión mínima al 80\% de personas adultas mayores sin pensión que generaría un aumento del gasto en pensiones situándolo en un $80 \%$ del PIB en 2030. El documento concluye que para hacer frente a los retos del envejecimiento poblacional es necesario conjugar un proceso de formalización laboral con un incremento de la demanda laboral, para así aumentar los ingresos y el PIB per cápita vía un crecimiento del producto y por ende incrementar las contribuciones a seguridad social en pensiones, que balancearían las finanzas del sistema pensional colombiano.

Este documento se divide en tres partes: i) Hechos estilizados del sistema de pensiones, las tendencias demográficas y el mercado laboral ii) simulación de escenarios y iii) conclusiones.

\section{HECHOS ESTILIZADOS}

\section{Sistema Pensional}

Para inicios de los años 90's, la cobertura del sistema de pensiones colombiano era bastante desalentadora. Según Helmsdorff (2007): "para el año de 1991 la cobertura del sistema pensional apenas alcanzaba el 25\% de la población". Por su parte Santa María et 
Gráfica No. 1 Total de pensionados sobre total de afiliados

Total de pensionados sobre total de afiliados

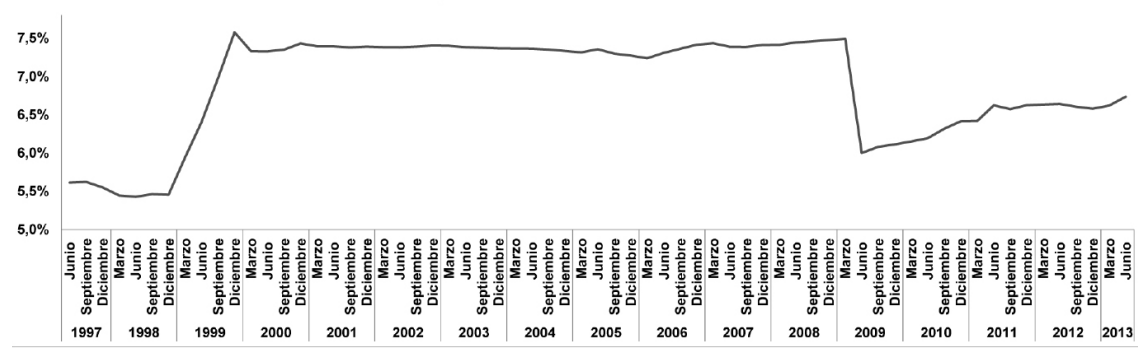

Fuente: Superintendencia Financiera de Colombia (2013)

Gráfica No. 2 Porcentaje de pensionados sobre la población mayor de 60 años

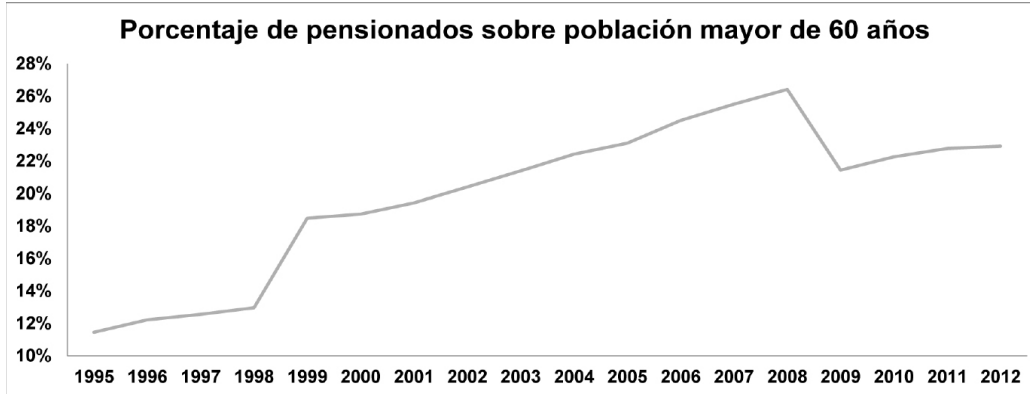

Fuente: Superintendencia Financiera de Colombia (2013) DANE (2013)

al (2010) afirma que antes de la reforma de 1993: "La tasa de cotización no seincrementó gradualmente como se había previsto desde el principio y el Estado incumplió su parte de la cotización. La diferencia entre las tasas de cotización programada y efectiva marcó, desde un inicio, el camino hacia la insostenibilidad financiera del SGP". En Colombia, la cobertura en pensiones de la población económicamente activa (PEA), ha sido siempre bastante baja. Como lo ilustran Reyes (2010), Acuña et al (2009), en 1973 la cobertura era de un 18\% de la PEA, mientras que en los años noventa superaba un poco más del $20 \%$, y en la actualidad el registro es de tan solo un $30 \%$ de la PEA.

En detalle, se puede determinar que la cobertura pensional del sistema sigue siendo aún baja por tres variables:
1. El número de pensionados con relación al número de afiliados al sistema es de tan solo el 7,3\%, lo cual es un indicador de la probabilidad de pensionarse. Esto indica que aproximadamente un 90\% de los afiliados al sistema no se pensionaran (Gráfica No.1).

2. El número de pensionados con relación a la población adulta mayor ${ }^{5}$ es del $26 \%$. Esto quiere decir que aproximadamente un $74 \%$ de los adultos mayores no se va a pensionar. Es importante considerar

5 De acuerdo al último censo del DANE (2005), la población adulta mayor se define como aquella población mayor igual a 65 años. Sin embargo, para esta investigación se toma en cuenta la población adulta mayor como aquella mayor igual a 60 años debido a que de acuerdo con la legislación colombiana la edad a pensionarse comienza a partir de los 60 años de edad. Siendo así, un rango de edad más adecuado para realizar las futuras mediciones del presente estudio. 


\section{Gráfica No 3 Porcentaje de ocupados que no cotizan a pensión 2007-2012}

Porcentaje de ocupados que no cotizan a pensión

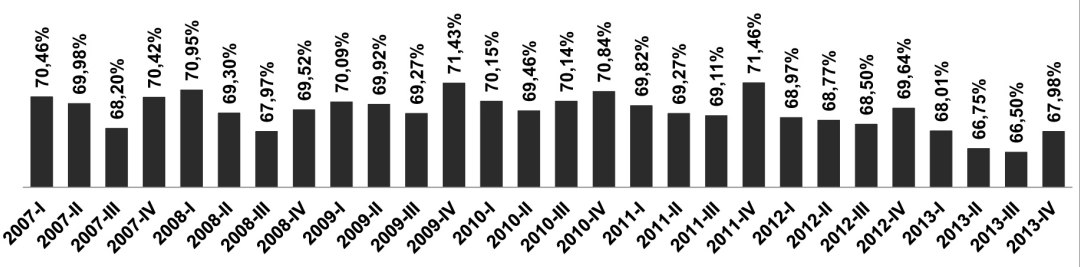

Fuente: DANE (2013) Cálculos: Autores

Gráfica 4 Relación cotizantes, total de afiliados y población económicamente activa

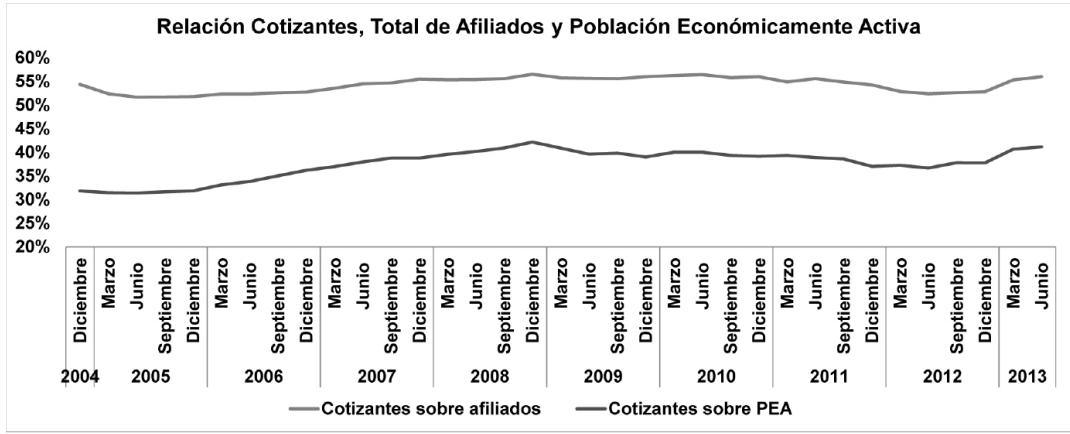

Fuente: Superintendencia Financiera de Colombia (2013), DANE (2013)

que con el paso del tiempo este grupo poblacional se incrementará, aumentando los desafíos en términos de pobreza, cuidado y desigualdad (Gráfica No.2).

3. El número de ocupados que cotiza para obtener una pensión es del 32\%, lo que potencialmente dejaría por fuera de una pensión para la vejez al 68\% de la fuerza laboral. De acuerdo con Santa María et al (2010) la baja cobertura afecta directamente a la población más pobre, mientras que los problemas fiscales siempre redundan en reducciones de cobertura entre esa misma población, $\mathrm{y}$, de manera importante, restringiendo gravementela disponibilidad derecursos para poner en marcha programas sociales focalizados también en esas poblaciones. Sin embargo, como se puede apreciar, el sistema ni siquiera alcanza a cubrir a la población más vulnerable, ya que no brinda instrumentos para incentivar y agrupar al sector informal que es donde se concentra la población de escasos recursos (Gráfica No 3). ${ }^{6}$

4. En términos de cotizaciones, si bien el 80\% de la PEA está afiliada al sistema de pensiones, tan solo el 40\% de la PEA es cotizante activa $^{7}$ en ambos regímenes a junio de 2013, pese a los incrementos que ha habido desde el 2004 del orden de 9,34 puntos porcentuales. Esta cifra es muy baja en términos internacionales. Es interesante resaltar

6 Vale la pena mencionar que la informalidad que se trabajó fue el número de Ocupados sin afiliación a la seguridad social, estrictamente sin afiliación a estimada por el DANE.

7 Según el Ministerio de Trabajo (2006) los afiliado activo cotizante, que son aquellos afiliados no pensionados que efectuaron la cotización obligatoria durante el mes para el cual se reporta, de manera periódica, correspondientes a aportes del mes que se paga, no a aportes de períodos en mora. 


\section{Gráfica No. 5 Distribución de las cotizaciones por SMMLV en el RAIS 2011-} 2012-2013

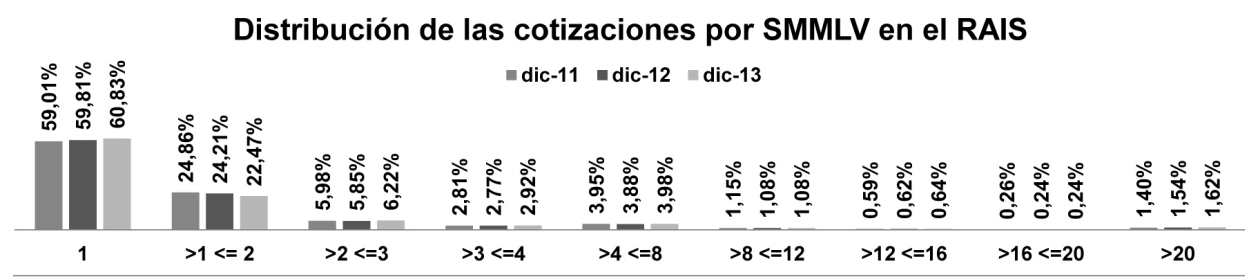

Fuente: Superintendencia Financiera de Colombia (2013)

que esta relación la tenía el sistema a mediados de los años 2000, pues según Sánchez (2008) el número de afiliados cotizantes y activos es de tan solo el $52 \%$ en el sistema pensional, considerando ambos regímenes (Gráfica No 4).

Al estudiar la distribución de las cotizaciones por Salarios Mínimos Mensuales Legales Vigentes (SMMLV), se encuentra que el grueso de la población cotizante realiza sus aportes para obtener una pensión de 1 SMMLV tanto en el Régimen de Prima Media (RPM) la cual es superior al $80 \%$, como en el Régimen de Ahorro Individual (RAIS) que está por encima del 90\%. En detalle, al estudiar la distribución de las cotizaciones por SMMLV en el RAIS, muestra que el 83\% de la población cotiza en promedio para obtener un SMMLV (59,88\%) o alcanzar entre uno o dos SMMLV (23,85\%) para los años 2001-20138 (Gráfica No 4).

Según Moreno \& Ortiz (2010) la cobertura real no se incrementó con las reformas mencionadas al comienzo de esta sección, pues si bien: "es cierto que la cobertura se aumenta al $70 \%$ de la PEA, tan solo el $40 \%$ del podrá llegar a tener una pensión en el régimen de ahorro individual". Esto, sin

8 Desafortunadamente, no se encontraron cifras más desagregadas para el Régimen de Prima Media, ni para el Régimen de Ahorro Individual para años anteriores a 2011. tener en cuenta que a 2008, el $92 \%$ de las personas entrevistadas por la encuesta continua de hogares, que recibían menos de un salario mínimo no se encontraban afiliados al sistema, con lo cual se corrobora que el sistema es excluyente para la población de bajos ingresos. De acuerdo a ello como lo mencionan Santa María \& Piraquive (2013): "apenas el 30\% de la población trabajadora cotiza efectivamente, sólo el 37\% de la población mayor de los 60 años tiene un beneficio pensional, y se prevé que esta va a descender cuando se apliquen los ajustes previstos".

5. Por último, en el tema financiero el gran desafío se centra en la indexación del rubro de pensiones en el presupuesto nacional, que para 2012 es de alrededor del $19 \%$ y de un 4,08\% del Producto Interno Bruto derivado del agotamiento de las reservas pensionales fruto del régimen de transición, del incumplimiento del incremento de las tasa de cotización por parte del Estado en años anteriores a la ley 100 de 1993 (como lo menciona Acuña et al (2009) y Santa María \& Piraquive (2013)). Esta relación entre el gasto pensional sobre el PIB, ha venido creciendo de manera gradual año a año, como se observa en la gráfica No 6, donde el déficit pasa a ser el 4,8\% del PIB en 2012, siendo el 4,03\% del PIB en 2010 y el 1,91\% en el 2002, cifras cercanas a los valores de estimados por 


\section{Gráfica No. 6 Evolución del gasto pensional total como porcentaje del PIB}

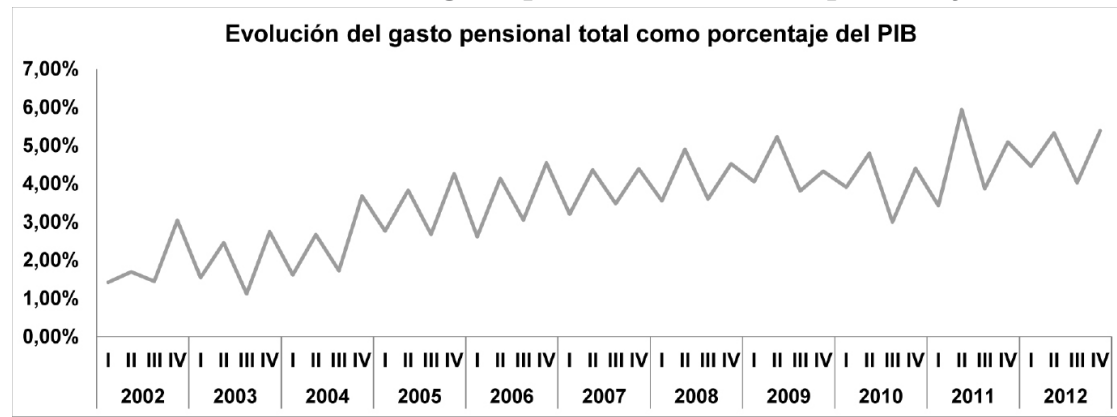

Fuente: Ministerio de Hacienda y Crédito Público (2013)

\section{Gráfica No. 7 Gasto en pensiones PGN 2000-2010}

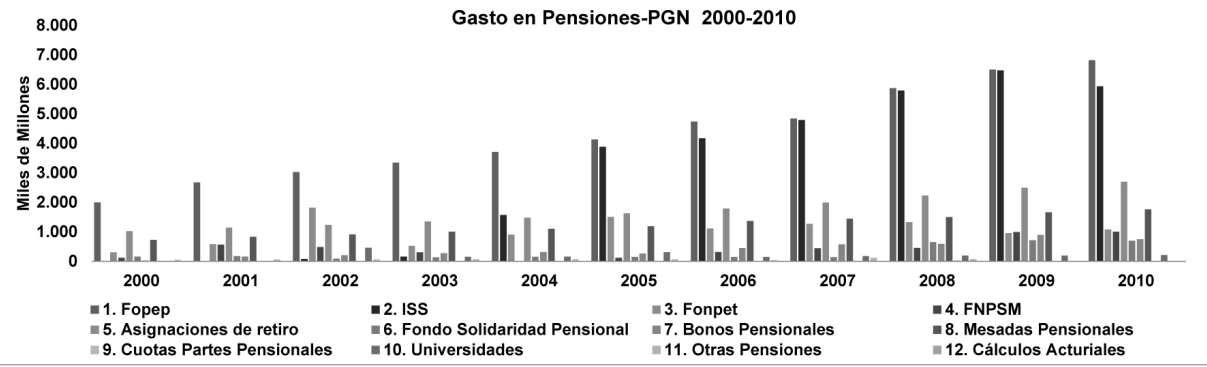

Fuente: Contraloría General de la República (2013)

Clavijo (1998), Clavijo et al (2013)', lo que implica según Rincón et al (2004) que: "las erogaciones por pensiones son la segunda causa más importante del desequilibrio fiscal del gobierno". Parra (2001) y Echeverry el at (2001) aseguran que para afrontar el déficit pensional no solo es necesario realizar reformas paramétricas al sistema, sino que debe de ir acompañado de la búsqueda de otras fuentes de financiamiento, como: "el re-direccionamiento de impuestos parafiscales, los recursos de la explotación de recursos naturales y el espectro electromagnético, utilización parcial de recursos de recursos del

9 No obstante, de acuerdo con Moreno \& Ortiz (2010) no existe una universalidad no universalidad $y$ consistencia de las cifras puesto que se han generado desde los años noventa diferentes estimaciones del pasivo pensional
Fondo de Estabilización Petrolera" (Gráfica No. 6) .

Vale la pena resaltar, que en el año de 2005, el gasto en pensiones tuvo un incremento significativo del 45,66\% respecto al año 2004 y del 89,17\% en relación al año 2003, producto del agotamiento de las reservas pensionales del Instituto de Seguro Social. Al respecto ver la gráfica No 7.

\section{Demografía}

De acuerdo con Lora (2008), el proceso de transición demográfica es el cambio que experimenta una población a partir de una situación inicial de altas tasas de natalidad y mortalidad a una situación final donde se dan bajas tasas de natalidad y mortalidad y aumenta la esperanza de vida. En este sentido, de acuerdo con Fló- 
Gráfica No. 8 Índice de dependencia vs Índice de Envejecimiento 1950-2100

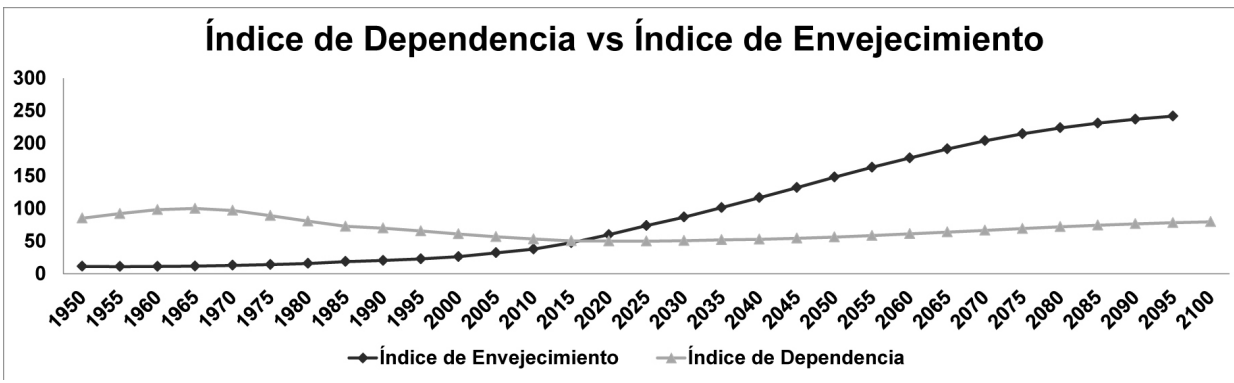

Fuente: UN Data (2010), Cálculos: Propios

rez (2000; Nostestein (1945)) "la transición demográfica asocia los cambios en las tasas de natalidad y mortalidad al proceso global de modernización". Según datos de Naciones Unidas (2012) la disminución en la tasa de natalidad promedio anual de $1,58 \%$ entre los años 1960 a 2020, previo a una reducción de la tasa de mortalidad promedio anual de 0,53\% para este periodo, acompañada de un incremento de la esperanza de vida que en promedio se proyecta de 76 años para los años entre el 2000 y 2050 y una disminución de la tasa de fecundidad proyectada para el 2020 de dos hijos por mujer en edad reproductiva, generaran un aumento de la población adulta mayor reflejado en un incremento de los índices de dependencia y envejecimiento. Es decir, en el futuro la composición población estará caracterizada por un incremento en la proporción de personas adultas mayores respecto a la población joven, proyectando un claro proceso de envejecimiento poblacional.

En consecuencia, para el siglo XXI, la población colombiana presentara cambios estructurales en su distribución poblacional, ya que como se observa en las pirámides de población ${ }^{10}$, se comenzara a repartir en proporciones más equitativas

10 Ver Anexo No 1 la población en los rangos de edad. En este sentido la pirámide poblacional, pasa de ser ancha en su base y estrecha en su punta para expandirse en los puntos medios de la pirámide hasta llegar a finales de siglo a una base más angosta que su punta, mostrando un acelerado proceso de envejecimiento poblacional. La población de la tercera edad será mayor que la población joven. Como lo menciona Lora (2008): "El estado de la transición y la velocidad con la que han ocurrido, determina el tamaño relativo de cada generación"

Evidentemente, el siglo XXI será el tiempo en el que la población colombiana envejezca puesto que el índice de envejecimiento presenta una tendencia creciente en el periodo, ya que por cada 100 personas menores de 65 años hay en promedio 15 personas mayores de 65 años en los años de 1950-2000, mientras que por cada 100 personas menores de 65 años hay en promedio 72 personas mayores de 65 años en los años de 20002050. Por último se proyecta un índice de envejecimiento de 203 para el periodo de 2050-2100 (Gráfica No. 8).

En concordancia con Fougère \& Mérette (1999) en un primer momento la transición demográfica contribuirá a un aumento del 
Gráfica No. 9 Relación índice de dependencia juvenil vs población mayor

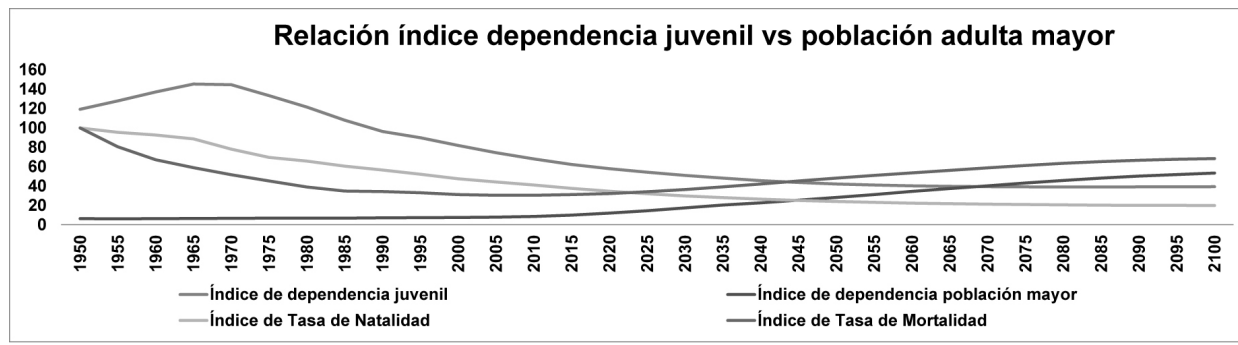

Fuente: UN Data (2010), Cálculos: Propios

ahorro pensional debido a que habrán más personas que cotizarán un seguro para su vejez fruto de un incremento de la tasa de natalidad. Sin embargo, con el pasar del tiempo el índice de dependencia juvenil disminuirá pero paralelamente el índice de dependencia de la población adulta mayor continuara incrementándose debido a que cuenta con el rezago del crecimiento demográfico fruto de un incremento previo en la tasa de natalidad. Lo anterior se traduce en que en un segundo momento el aumento de la proporción del índice de dependencia de la población adulta mayor comenzara a incrementarse, es decir, el envejecimiento poblacional ejercerá una fuerte presión para que el ahorro pensional disminuya. Para el caso colombiano, lo anterior se evidencia en la gráfica No 9.

Más allá de medir cuantas personas adultas mayores existirán en relación a las personas menores a sesenta años, el envejecimiento poblacional demanda una serie de transformaciones y políticas públicas en lo referente a la atención en pensiones, salud, regulación, cultura, integración a las comunidades de forma productiva, movilidad, infraestructura y cuidado ${ }^{11}$.

11 Para profundizar en los impactos del envejecimiento profundizar en: Torres \& Peláez (2012), CEPAL (2009), Vial (2013), Salazar (2007), UNFPA (2012), Schmidt (1999).
Como lo enfatizan Börch-Supan \& Ludwig (2009), Narciso (2010), Ito \& Tabata (2010), Domeij \& Floden (2003) el incremento en la proporción de población adulta mayor frente a la reducción de jóvenes puede generar dos efectos: i) Un aumento en la tasa impositiva en seguridad social, reduciendo los ingresos de los hogares; ii) Un descenso en el ahorro de la economía producto de una disminución en la fuerza de trabajo. Por tanto como lo exponen Higgins \& Williamson (1996), Higgins (1998), Turner et al (1998), Supan et al (2001), para expandir el capital acumulado de los ahorros de la población, se recurre al flujo de capitales internacionales en búsqueda de mayores tasas de retorno que brinden una mayor rentabilidad. En este orden de ideas, Poterba (2001), afirma que una sociedad envejecida necesita más capital gracias a que este sustituye cada vez más la mano de obra. Así, un incremento en la demanda de capital real aumenta los retornos de capital. Sin embargo este proceso estará marcado por la inestabilidad propia de los mercados financieros globales.

En resumen, en concordancia con Díaz \& Jiménez (2001) el envejecimiento poblacional, genera repercusiones en materia económica, perjudicando la estabilidad financiera de los sistemas de seguridad social, en específico, el sistema 
de pensiones, puesto que incrementa el índice de dependencia demográfica gracias a que aumenta el número de personas de población mayor por cotizante. En otras palabras, en un régimen de prima media: "son los cotizantes de un periodo los que con sus aportaciones financian las prestaciones que reciben los jubilados de ese mismo período". Al analizar la coyuntura de la transición demográfica colombiana con su consecuente envejecimiento poblacional y su relación con el sistema de pensiones, como lo menciona Urdinola (2011) y Torres \& Peláez (2012) la cobertura en pensiones es tan solo del $25 \%$ de la fuerza laboral lo cual ejercerá una fuerte presión en términos fiscales puesto que eventualmente por medio de recursos públicos se deberá atender a una población envejecida sin protección institucional.

Por último, como lo afirma Vial (2013) en términos macroeconómicos se incrementa el gasto público ligado a las presiones de demanda de servicios en seguridad social, que se traducen en aumentos de impuestos y en un alza en la prima de riesgo del financiamiento fiscal causando más inestabilidad macroeconómica, lo cual se verá evidenciado en mayor gasto en consumo y transferencias sociales reduciendo así el ahorro y la inversión pública en otras áreas. En detalle, el proceso de envejecimiento implica presiones en el sistema de pensiones: "por el aumento de las personas en edad de jubilar y, especialmente, de la relación entre los que reciben beneficios de jubilación respecto de los que aportan, algo que es crítico para sistemas de reparto".

Por su parte en los sistemas de ahorro individual, como lo expone Rodríguez et al (1992) el envejecimiento significaría coacciones en términos de equidad puesto que: "El sistema tiende a ser fuertemente selec- tivo y sesga los beneficios del desarrollo a favor de la población de altos ingresos". Es decir que debido a su: "relación directa beneficios-cotización", deja sin seguro de vejez a la población más vulnerable ya que elimina el concepto de solidaridad directa entre los quintiles de ingresos más altos con los quintiles de ingresos más bajos. Adicionalmente, como lo menciona Antolin (2001) el envejecimiento poblacional también afectará a las pensiones privadas mediante el impacto en los mercados financieros, en detalle, en la diversificación de los portafolios y en los retornos esperados de la inversión. Al estar el portafolio basado en los ciclos de vida ${ }^{12}$, podrá incrementar su proporción de inversión en mercados financieros en relación al incremento promedio de edad de la población en edad de trabajar. Como consecuencia, los retornos de dichas inversiones podrán caer ligeramente mientras el riesgo disminuye. Al mismo tiempo, el aumento en la expectativa de vida también incrementara directamente la cantidad de ahorro necesario para conservar cierto estándar de vida al momento de la jubilación.

\section{Mercado Laboral}

En el tema laboral, son dos los cuellos de botella que relacionan al mercado de trabajo con el sistema de pensiones, la informalidad y la baja productividad. En primer lugar, al clasificar el número de ocupados se encuentra la cantidad de

\footnotetext{
12 Según Antolin (2001) Considerando la hipótesis de ciclo de vida entendida que las personas adultas mayores tienen una mayor propensión al consumo a partir de su ingreso, ya que en su vida laboral ahorraron para asegurar un ingreso-seguro de vejez, por consiguiente una proporción mayor de personas mayores con respecto a las personas en edad de trabajar reducirá el ahorro. Por otro lado, una reducción del ahorro demandará una tasa menor de acumulación de capital que necesitará proveer una disminución en la fuerza laboral.
} 
Gráfica No. 10 Clasificación del número de ocupados

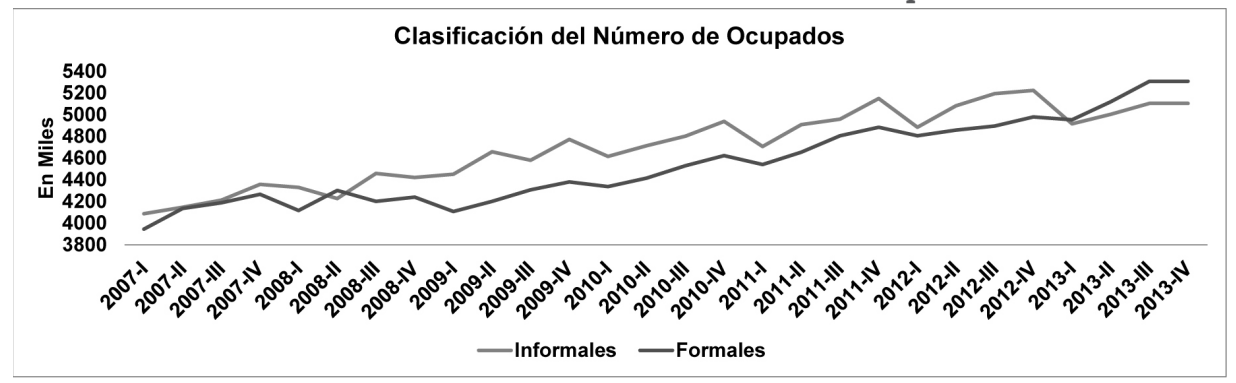

Fuente: DANE (2013) Cálculos Autores

Gráfica No. 11 Tasa de Informalidad 2001-2013

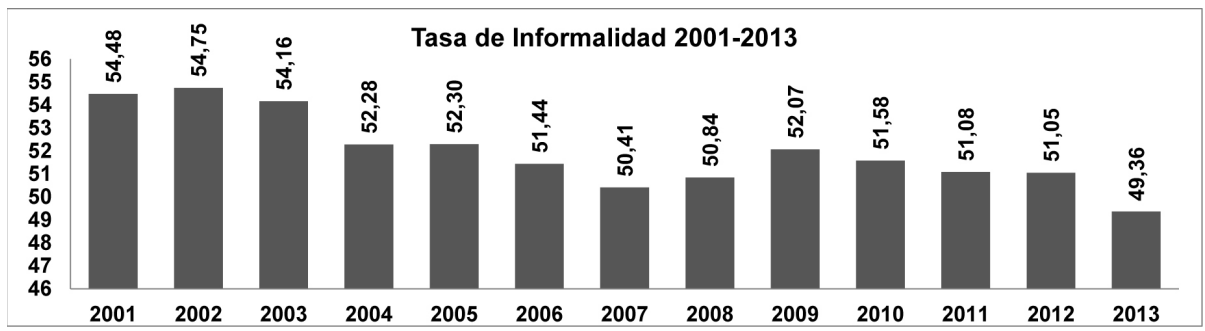

Fuente: DANE (2013) Cálculos Autores

personas con empleo formal e informal ${ }^{13}$ con que cuenta la economía. Las cifras muestran una tendencia creciente para ambos grupos, sin embargo el grupo de los formales registró un mayor incremento del orden del $26,76 \%$ frente al aumento del 21,20\% del primer grupo, entre el 2007-IV al 2013-IV (Gráfica No.10).

Como lo menciona Ayala \& Acosta (2002), Bernal (2009), Flórez (2002) la informalidad no solo implica una baja productividad, sino también ausencia de cotización pues este sector no realiza aportes a seguridad social, careciendo de un seguro de salud y de vejez. Por su parte Uribe (2002) menciona "el carácter informal de las relaciones laborales, que es generalizado en la mayoría de los países de la región, señala, a la vez, el límite de la expansión de

13 Vale la pena mencionar que la informalidad que se trabajó fue la proporción de informalidad según tamaño de empresa hasta 10 y hasta 5 trabajadores estimada por el DANE. la cobertura y las deficiencias en el diseño del sistema". ${ }^{14}$

De acuerdo con Arango \& Hamann (2012), Arango et al (2013) la baja productividad laboral de la mano de obra no calificada se evidencia en el tamaño del sector informal siendo una de las principales características del mercado laboral colombiano. Según estimaciones de Cespedes et al (2011): "el 39.2\% de los trabajadores colombianos contribuyen con tres cuartas partes del PIB, lo cual implica que la productividad del sector formal es 6.7 veces la del informal", reafirmando la baja productividad laboral que tiene del mercado de trabajo colombiano. El efecto de la informalidad laboral tendrá sus efectos en el sistema de seguridad social en pensiones, según López (2012) debido a que "los informales no cotizan para pensión, en el futuro el Estado colombiano tendrá a su cargo el

14 Datos provisionales a 2013. 
sostenimiento de una masa importante de personas que no tomaron ninguna medida para sostenerse en su vejez".

Gran parte de la literatura económica ${ }^{15}$, relaciona una disminución de la informalidad debido a la liberación del mercado laboral manifestado en la reducción de los costos no laborales. Como lo sustenta Bernal \& Cárdenas (2004) y Bustamante (2006) los costos laborales propician la reducción de la generación del empleo viéndose sus efectos en el mercado laboral y en los sistemas de pensiones y de salud. "Dado el incremento de ellos probablemente el efecto en la mano de obra, salud y pensiones ha sido una disminución de la generación de empleo".

Si bien al incrementar el número de personas que realizarían sus cotizaciones con una tasa de fidelidad alta al ser formales, estas cotizaciones serían bajas debido a que están ligadas a bajos ingresos producto de las características de un trabajo informal con baja productividad. En este sentido es necesario que el Gobierno realice esfuerzos (inversiones) en incrementar la productividad laboral y así aumentar las contribuciones a los regímenes pensionales como lo señala OECD (2011); es necesaria la elaboración de: "políticas de protección social [que] deben ser diseñadas en conjunto con un cuadro de las instituciones sociales, laborales y macroeconómicas adecuadas". Igualmente como lo demuestra Jiménez-Ridruejo (2005) la productividad laboral incrementa el número de cotizaciones para pensiones vía un aumento de los salarios. Adicionalmente, como lo expresa Sandoval (2012) "la informalidad no puede explicarse simplemente como un problema derivado de los costos asociados al ingreso al mercado

15 Para mayor profundización en el tema véase Santa María et al (2010), Bustamante (2006), Mondragón et al (2010), Cárdenas \& Bernal (2004), Sandoval (2013). formal sino que es parte de un problema estructural de la economía nacional que está relacionado con las características de su estructura productiva". A pesar de que la economía colombiana ha presentado crecimientos importantes en los últimos años, del orden de $4 \%$, retornando a los niveles registrados en los años 60's y 70's, la productividad total de los factores (PTF) no ha recuperado el nivel presentado bajo el modelo de industrialización dirigida por el Estado como lo atestiguan Loayza et al (2004) Echeverry \& Santamaría (2004), y treinta años después no ha recuperado su nivel.El problema es principalmente atribuido a factores estructurales, lo cual como lo señala Arango \& Hamann (2012) coloca "en evidencia el bajo nivel de productividad de la mano de obra".

Concordantemente, la OECD (2011), y Moreno \& Ortiz (2010) establecen que la relación entre la informalidad laboral y los sistemas de protección social crea un círculo vicioso, en el cual la masa de trabajadores informales debilita los sistemas de contribución de los mismos, puesto que la baja productividad ligada a los bajos ingresos y la inestabilidad del sector no permiten conservar una sostenibilidad en los aportes ni una fidelización al sistema respectivamente. Estudios como los de Rada (2009), CondeRuiz \& Alonso (2006), Jimeno (2002), Alonso \& Herce (2003), afirman que existe una relación directa entre la productividad laboral y las pensiones, puesto que CondeRuiz \& Alonso (2006): "los incrementos en productividad se trasladan a los salarios y como sabemos las pensiones dependen de los salarios durante la vida laboral", incrementando las cotizaciones del sistema contribuyendo a la mejoría de la sostenibilidad del mismo.

Es más, respecto al desafío de la sostenibilidad financiera del sistema pensional que 
básicamente radica en las contribuciones de los afiliados activos, como lo menciona Clavijo (2009): "En ambos sistemas, las contribuciones necesitan mejorar en densidad, es decir incrementar la formalidad laboral, y en cantidad, en otras palabras aumentar el beneficio por acción de las empresas que será canalizado a través del sistema". Siguiendo esta línea, Reyes (2010) concluye que una de las vías que tiene el Gobierno Nacional para el sostenimiento del sistema de seguridad social en pensiones es apostarle a la formalidad laboral acompañada de un incremento en la productividad puesto que: "un aumento en la proporción de personas empleadas supondría mayor número de aportantes al sistema disminuyendo el peso sobre el fisco nacional. Así mismo, incrementando la productividad laboral media, reflejada en el PIB por trabajador, se conseguiría contrarrestar el efecto negativo del factor demográfico sobre el gasto en pensiones".

En este sentido, el impacto del envejecimiento poblacional dependerá fundamentalmente de la productividad y de la posibilidad de fortalecer el régimen de prima media pues ya que como afirma Reyes (2010): "si la transferencia intergeneracional de ingresos se desliga de la dependencia que la tercera edad tiene del mercado de trabajo formal, y se vincula a la producción general que el mercado de trabajo genera, las nuevas proporciones demográficas dejan de representar un "desequilibrio" significativo para el financiamiento del sistema previsional". En la misma línea la Cepal (2009) concluye que: "las relaciones de dependencia tenderán a disminuir por lo menos durante un cierto lapso, por lo que la mayoría de los países de la región tendrá ante sí la posibilidad de ampliar el potencial productivo y prepararse para la fase final de transición demográfica caracterizada por el incremento relativo de la población de mayor edad".

\section{Análisis Metodológico}

Tomando como referencia lo expuesto anteriormente y la literatura económica que ha trabajo el tema donde se destacan los estudios de Santa María \& Piraquive (2013), López \& Lasso (2012), Arias \& Mendoza (2009), Reyes (2010), entre otros, para esta investigación se toma como base el modelo planteado por Boldrin et al (1999), que simula la carga pensional de los países de la Unión Europea para los años de 1995 a 2050, y trabajado por Jimeno (2002) para el caso español y por Moreno \& Ortiz (2010) para el caso colombiano. En este modelo el gasto público en pensiones como porcentaje del Producto Interno Bruto es explicado por cuatro relaciones. La primera relación es la cobertura pensional, la segunda relación es el índice de dependencia demográfica, la tercera relación es el funcionamiento del mercado laboral y la cuarta relación es uno de los factores institucionales del sistema, a saber: el nivel del valor de la pensión media con respecto a la productividad del trabajo. El modelo puede ser expresado de la siguiente manera:
Sin embargo, como el objetivo de esta investigación es conocer el efecto de la formalización laboral en el gasto público en pensiones, se in- trodujo esta variable al modelo realizando una modificación a la relación del mercado laboral, dando como resultado la siguiente expresión:

\footnotetext{
Gasto en Pesiones

Producto Interno Bruto

$=\frac{\text { Población }>60}{\text { Población entre } 15-60} \frac{\text { Número de pensionados }}{\text { Población }>65} \frac{\text { Pensión Media }}{\text { Productividad laboral }} \frac{\text { (Formales)(CrecimientoEconómico) }}{\text { Ocupados }}$
} 
En términos individuales, las variable dependiente del modelo tiene como fuente las publicaciones del Ministerio de Hacienda y Crédito Públicodel presupuesto del Gobierno Nacional Central, tanto el rubro de gasto en pensiones como el Producto Interno Bruto, pero estos son publicados en términos nominales, por lo tanto para comprobar el verdadero efecto de las variables explicativas del modelo en él, se deflactan las cifras a fin de expresarlas en términos nominales ${ }^{16}$.

En cuanto a las cuatro variables independientes del modelo estas representan una dinámica especifica. El primer cociente representa el índice de dependencia demográfica, mide la proporción de adultos mayores sobre la edad productiva reuniendo el envejecimiento poblacional ya que toma el efecto de la reducción de las tasa de natalidad y mortalidad e incremento de la esperanza de vida en la analogía la población adulta mayor con respecto a la fuerza laboral.

Por su parte los dos siguientes cocientes representan el funcionamiento del sistema pensional colombiano y específicamente los cuellos de botella mencionados anteriormente: la baja cobertura y la baja fidelización del sistema. El Factor Institucional 1, correspondiente al número de pensionados sobre la población adulta mayor encarna la relación entre el número de pensionados de ambos regímenes. Este Factor Institucional 1 determina la elegibilidad para recibir la pensión y contempla aspectos como las semanas mínimas de

16 Para mayor profundización en el tema véase Moreno \& Ortiz (2010).

$17 \mathrm{Al}$ considerar un modelo exponencial beta, la ecuación a estimar es la siguiente: cotización y la edad de retiro con lo cual se evidencia el porcentaje de adultos mayores que gozan de una pensión. Por su parte el Factor Institucional 2, está relacionado con la proporción entre la pensión promedio expresada en términos de salarios mínimos sobre la productividad laboral, entendida esta según Sargent \& Rodríguez (2001) como el valor de la producción por unidad de trabajo, es decir, cuanto aporta el trabajo a la productividad total de los factores en la economía. Este cociente incorpora aspectos como la historia laboral del trabajador, sus ingresos y sus contribuciones al sistema, siendo una aproximación a la fidelidad del mismo.

Por último, la variable del mercado laboral incorpora los elementos del empleo formal y la contribución de estos al crecimiento económico, así capta las tenciones del mercado de trabajo y las políticas de mercado laboral ligadas con el crecimiento económico, midiendo la producción de los trabajadores formales con respecto al crecimiento económico.

En consecuencia para determinar cuál será el impacto de las dinámicas del sistema pensional, demográficas y del mercado laboral en el gasto público en pensiones, se proyecta el comportamiento individual de las variables descritas anteriormente al año 2050, con el fin de establecer un escenario base fundado en las tendencias presentadas para cada una de las variables, mediante la estimación de un modelo beta exponencial que toma en cuenta los rango entre 0 y 1 de la variable independiente para conservarlos en esta categoría en los pronósticos a realizar ${ }^{17}$. Por ende, considerando las tendencias presentadas 
por las variables, el gasto pensional presentaría una disminución radical entre los años de 2014 a 2020, alimentado por un descenso de la población de pensionados puesto que de acuerdo con el Ministerio de Trabajo (2013) un 90\% de los trabajadores no logran pensionarse, ya sea porque se encuentran afuera del sistema, como es el caso de los trabajadores informales, o por las bajas tasas de fidelización que existen en el ciclo de vida, producto de la inestabilidad del mercado laboral, tal y como lo reflejan los trabajos de los trabajos de Clavijo (2012), López \& Lasso (2012), Ministerio de Trabajo (2013), entre otros. Si bien se cuenta con una población que realiza algunos aportes durante su vida laboral, estos no son los suficientes para obtener una pensión al finalizar su ciclo de vida, por consiguiente tan solo un $33 \%$ de los ocupados cotiza para obtener una pensión sin agregarle la estadística agravante del Ministerio de Trabajo según la cual tan solo $8 \%$ de estos cotizantes llegan a pensionarse (Gráfica No.12).

\section{Gráfica No 12 Escenario Base}

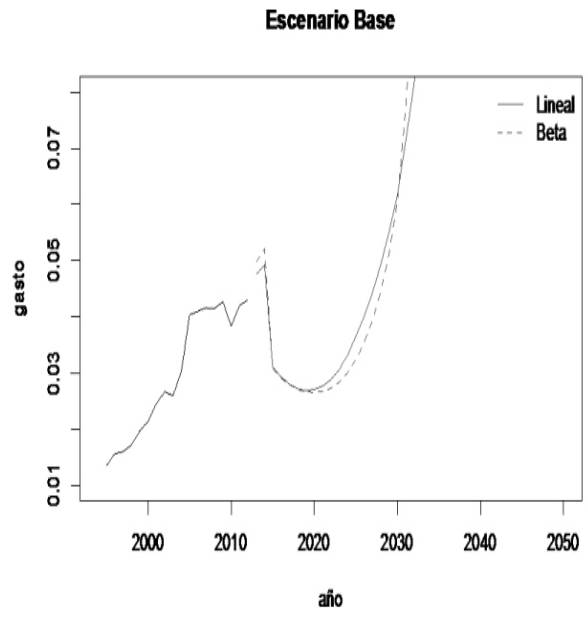

Fuente \& Cálculos: Autores
Sin embargo, al estudiar detalladamente las proyecciones de población de Naciones Unidas (2012), se observa un aumento de la población mayor de 60 años del 4,67\% promedioanual entreel 2015a 2030, la cual es la tasa más alta que registra el país en la muestra entre 1990-2100. Esto significa que en este periodo habrá más personas mayores de 60 años, que guardando las tendencias actuales, y tan solo un $20 \%$ de ellas tendrán un seguro para la vejez. El restante $80 \%$ de la población mayor, que serán en promedio unos 5 millones y medio de personas entre 2015-2030, representando el $11 \%$ de la población proyectada, demandarán un seguro asistencial para la vejez puesto que por fricciones del mercado laboral no lograron acceder a una pensión contributiva, por lo tanto es responsabilidad de un Estado social de derecho que busca contribuir a la reducción de la pobreza e indigencia en la sociedad, intervenir para subsidiar este seguro de vejez viéndose reflejado en un drástico incremento en el gasto en pensiones como porcentaje del PIB de alrededor 6\% en 2030. De hecho, estos seguros para la vejez ya están siendo acogidos por el Gobierno Nacional por medio de los programas de Benéficos Económicos Periódicos (BEPS) y Colombia Mayor. Sí bien, estos programas buscan incrementar la cobertura real pensional que realiza periódicamente aportes al sistema oscila en $42 \%$ de la PEA, que un debate abierto en cuanto a la suficiencia del seguro de pensión al que accederá esta población.

En este orden de ideas, bajo el escenario hipotético de un incremento en el número de empleados formales en un 1\% anual, considerando las dinámicas de dependencia demográfica descritas en el anterior escenario, el impacto en el gasto en pensiones sería similar al 


\section{Grafica No 13 Escenario 2: Aumento de empleados formales \\ Escenario 2}

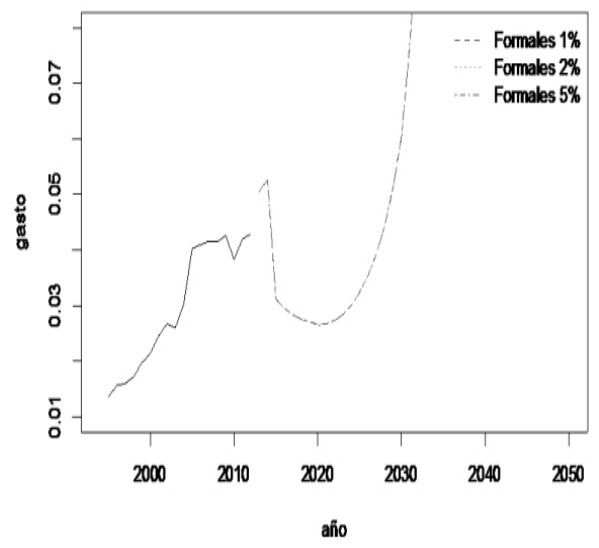

Fuente \& Cálculos: Autores

escenario base, incluso sí los formales incrementarán al $2 \%$ ó al $5 \%$ anual en las perspectivas más optimistas, la tendencia del gasto sería similar al escenario preliminar. Efectivamente, un aumento de los formales incrementaría la cobertura pensional, pero en términos reales los aportes no aumentarían, ya que el proceso de formalización se está dando en actividades poco productivas que no generan una multiplicación del producto y por ende de los ingresos, teniendo en cuenta que las actividades económicas productivas en su gran mayoría son formales. Esto generaría que los trabajadores ya formalizados realicen contribuciones al sistema pero que éstas no sean lo suficientes para generar el ahorro necesario para garantizar un seguro en la vejez. Es importante resaltar entonces que según nuestro modelo, la sola formalidad no disminuiría el gasto en pensiones como porcentaje del PIB, dado el efecto de dependencia demográfica anteriormente descrito, como se muestra en la gráfica No 13.
Por otra parte en el escenario 3, un aumento de la productividad laboral del $1 \%$ anual, tendría un efecto similar en el gasto pensional como porcentaje del PIB presentado en los escenarios anteriores. Sin embargo, sí se llegase a incrementar la productividad laboral en un $2 \%$ anual, el gasto en pensiones continuaría disminuyendo en más dos puntos porcentuales del PIB a partir del 2016, suavizando el incremento del mismo desde el 2023. Igualmente, en un caso más positivista sí se llegase a aumentar la productividad laboral en un 5\% anual, eventualmente se reducirá el gasto en pensiones como porcentaje del PIB, en casi más de una unidad porcentual, suavizando el aumento del mismo desde el 2030, como se observa en la gráfica No 14.

Ciertamente, un incremento en la productividad laboral causaría un incremento de la producción ya que el factor de capital humano tendía mayores capacidades y habilidades que harían incrementar el producto, que por ende aumentaría los ingresos laborales de los trabajadores incrementando así los aportantes pensionales de los mismos lo cual conduciría a una reducción en el gasto pensional y se ampliarían las probabilidades de pensión de los trabajadores. Debido a que las contribuciones que los trabajadores realicen al sistema serán mayores, acrecentando la probabilidad de que éstas sean lo suficientes para generar el ahorro necesario para garantizar un seguro en la vejez, como lo respalda Kathuria et al (2011) y Balkrishman (2004).

Por último, se contempla un escenario de aproximación asistencialista donde el Estado entra a subsidiar una pensión de un SMMLV a la población no pensionada, que según las proyecciones de la tendencia de la variable, se sitúa alrededor del $80 \%$ de la población adulta mayor a 2050; con el fin de 
Gráfica No 14 Escenario 3: Aumento de la productividad

Escenario 3

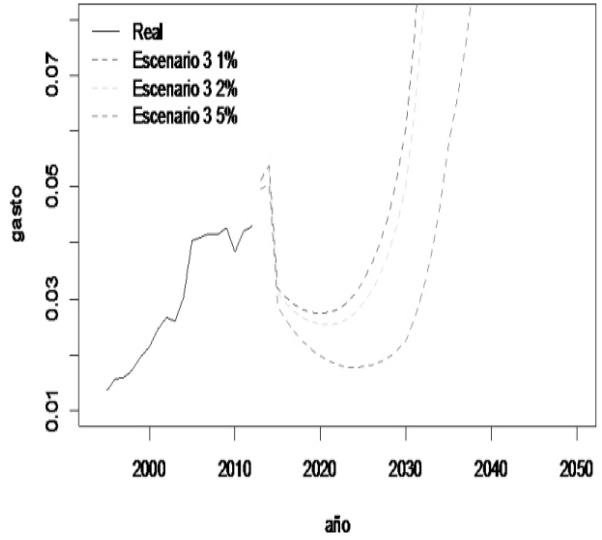

Fuente \& Cálculos: Autores
Gráfica No 15 Escenario 4: Aproximación asistencialista

Escenario 4

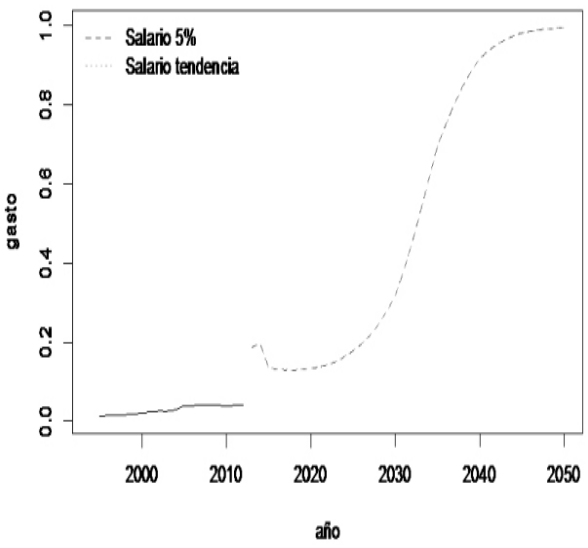

Fuente \& Cálculos: Autores dar un seguro en la vejez a esta población vulnerable ya que por sus condiciones físicas difícilmente podrá ser parte de la fuerza laboral activa. En efecto, para estudiar una aproximación del impacto asistencialista la ecuación planteada para los tres anteriores escenarios cambaría de la siguiente manera:

\section{Gasto en Pesiones}

Producto Interno Bruto

$=\frac{\text { Población }>60}{\text { Población entre } 15-60} \frac{\text { Número de No Pensionados Salario Mínimo Medio }}{\text { Población }>65} \frac{\text { (Formales) (CrecimientoEconómico) }}{\text { Productividad laboral }}(3)$

Las variables ligadas al sistema pensional cambiarían. La variable Institucional 1 tendría un cambio en el numerador ya que pasaría de ser el número de pensionados a ser el número de no pensionados, a su vez la variable Institucional 2 asumiría un cambio en el numerador puesto que pasaría de ser la pensión media por el salario mínimo medio, proyectado a $2050^{18}$.

Como se observa en la gráfica No 15, el efecto de la dinámica de demográfica sigue presente en este este escenario, pero a partir del año 2016 el gasto en pensiones como porcentaje del PIB tendría un explosivo incremento llegando a cifras del $90 \%$ del PIB para los años de 2040, teniendo

$18 \mathrm{Al}$ realizar la proyección se consideró el supuesto de una inflación constante de alrededor del $3 \%$ anual. en cuenta niveles de productividad y formalidad proyectados en la tendencia presentada entre 1990 a la actualidad. En este aspecto, el incremento de la población adulta mayor no solo representa un aumento de la demanda de los servicios en seguridad social en pensiones, sino también un incremento de la demanda de servicios en salud, en servicios de la economía del cuidado y gerontológicos, los cuales la sociedad colombiana de hoy no está preparada para brindarlos. Por tanto el envejecimiento poblacional ligado a los incrementos de los índices de dependencia demográficos es un desafío no solo en el tema del financiamiento del sistema pensional sino también lo es a nivel de la sociedad ligado a temas de servicios de salud y gerontológicos que garanticen la 
calidad de vida del grupo de población de adultos mayores.

\section{CONCLUSIONES}

Un aumento de la población adulta mayor significará un incremento del gasto público en pensiones como se demostró en esta investigación ya que en términos tangibles se observa un crecimiento del 4,67\% promedio anual de la población mayor de 60 años entre el 2015 a 2030, la cual es la tasa más alta que registra el país en la muestra entre 1990-2100. En otras palabras, si se conservan las tenencias actuales para este periodo tan solo un $20 \%$ de la población mayor de 60 años accederá a una pensión, mientras que el faltante $80 \%$ de este grupo poblacional, unos 5 millones y medio de personas en promedio entre 2015-2030, demandarán una ayuda asistencial para la vejez dado que por las condiciones del mercado laboral no lograran acceder a una pensión contributiva. En este punto es donde el Estado Colombiano en tanto Estado Social de Derecho, y en aras de disminuir los índices de pobreza e indigencia deberá actuar vía gasto público. La exclusión social de los adultos mayores presionara al Gobierno para que efectué planes asistencialistas a este conjunto de la población vulnerable, garantizándoles una ayuda asistencial contra la vejez, la cual, de hecho, ya está otorgando con la implementación de programas asistencialistas como lo son los Beneficios Periódicos Económicos (BEPS) y Colombia Mayor. No obstante queda en tela de juicio la suficiencia del subsidio económico entregada por estos programas y la cuantificación de sus impactos financieros en el gasto en pensiones del presupuesto nacional.

Un incremento en la formalización laboral puede significar una expansión en la cobertura del sistema ya que más trabajadores estarían vinculados formalmente al mercado laboral aumentado la probabilidad de que realicen contribuciones periódicas a la seguridad social en pensiones y de este modo se podría cambiar la tendencia creciente del gasto público en pensiones, fruto de los programas asistencialistas. El problema radica en saber si los trabajadores colombianos estarán en condiciones de realizar aportes al sistema pensional para obtener una pensión de manera sistemática y suficiente. Sin embargo, la sola formalización no es suficiente para contrarrestar el efecto del envejecimiento poblacional ligado al tema asistencialista. Un aumento en la tasa de formalización, no significa un incremento en las contribuciones al sistema, pues sí bien estas se realizarían periódicamente, el monto de estas no sería suficiente para acceder a una pensión. Estas cotizaciones de los nuevos formales, serían bajas debido a que están ligadas a bajos ingresos producto del rezago de un trabajo informal con baja productividad, teniendo en cuenta que el $60 \%$ de los asalariados cotiza para un Salario Mínimo. El problema es la estructura productiva colombiana. La baja productividad no permite extender la competitividad laboral estancando el crecimiento de la demanda agregada. Un aumento en la productividad es la única manera de generar incrementos importantes en el Producto Interno Bruto que impliquen un mayor PIB e ingreso per cápita y que por ende permitan incrementos reales en las cotizaciones, superiores a los propios de aquellos que ganan un Salario Mínimo, contribuyendo así a equilibrar las finanzas del sistema, como se demostró en el escenario 3 de este análisis, en el cual se proyecta una reducción en el gasto público en pensiones como porcentaje del PIB dado un incremento de la productividad.

Las últimas recomendaciones al sistema pensional colombiano de la OECD (2015) 
han vuelto a privilegiar las reformas paramétricas. La ampliación de la cobertura pasa por expandir los programas asistencialistas tipo beneficios económicos periódicos y Colombia mayor. Estos programas no hacen parte de un sistema de pensiones debido a que por mandato constitucional ningún colombiano podría gozar de una pensión menor al salario mínimo. Adicionalmente, si fuese el caso de una reforma constitucional, el tema de la suficiencia del ingreso pensional que no fue tratado en esta investigación- sería un punto importante a considerar.

Complementariamente, la recomendación de aumentar los requerimientos en los parámetros de la edad, igualdad de la edad de retiro entre hombres y mujeres, e incrementar las semanas de cotizaciones, tendrían un incremento marginal decreciente en gasto debido al envejecimiento poblacional y la informalidad. Como se ha venido desarrollando a lo largo del documento, el envejecimiento poblacional se traduce en una menor tasa de remplazo que conllevaría a menores cotizaciones en el largo plazo, mientras que el segundo factor no garantiza una sostenibilidad financiera en las cotizaciones sin tratar el tema de la audiencia de los ingresos, de nuevo. Es más como lo expone la misma OECD (2015) "Las proyecciones del radio de dependencia poblacional incrementaran sustancialmente, como resultado del presente envejecimiento poblacional de la población joven. La situación se empeora considerablemente cuando el radio de dependencia económica, que compara el número de personas de edad de 65 años y más con los empleados-que son potencialmente contribuyentes al sistema, incluyendo trabajadores informales. Como consecuencia de este envejecimiento poblacional, surge la necesidad de un soporte asistencial de ingreso para la población de edad, incrementado así significativamente las finanzas públicas".

Ciertamente hasta la fecha, las reformas se han centrado en temas paramétricos que han causado alivios financieros a la situación del corto plazo, a costa de los derechos de los trabajadores. Las futuras reformas deben abordar los problemas estructurales en tres vías: i) Proponer incentivos de cotización al régimen de pensiones en pro de promover la inclusión de más personas al régimen de prima media teniendo en cuenta los factores de informalidad y baja productividad del mercado laboral que es una imagen en el tiempo del sistema pensional. ii) Crear instrumentos de ampliación de cobertura para contrarrestar el fenómeno de transición demográfica y envejecimiento poblacional que causara un incrementa en la demanda de servicio de seguridad social en pensiones, iii) Diseñar una política integral en pro a incrementar la productividad laboral que como lo menciona Sargent \& Rodríguez (2001) "un aumento en la formación de capital humano e innovación, el cual incrementa la productividad y el producto el cual de nuevo en un segundo efecto, aumentaría el capital humano y la innovación, creado un circulo virtuoso", en aras a aumentar la producto que repercutirá en un crecimiento del PIB e ingreso per cápita y por ende de las cotizaciones a pensiones, ya siendo estos trabajadores formales, como lo menciona Romer (1990) "la clave del crecimiento y desarrollo sostenible es una adecuación del stock del capital humano". En últimas como lo expresa Echeverry et al (2001) "La dinámica demográfica define el número de cotizantes, el cual incide en la cantidad de pensionados a futuro, de acuerdo con las condiciones del mercado laboral (empleo, desempleo, informalidad). Una vez definidas las personas en el 
sistema, importa el papel que desempeñan, bien sea como cotizantes o pensionados, y el tiempo durante el cual permanecen en dichos roles. El periodo de disfrute de la pensión deberá estar en concordancia con el período de cotización o afiliación. En la medida que una persona permanezca más tiempo cotizando podría tener derecho a un mayor periodo paragozar de su mesada". A lo que complementan Santa María \& Piraquive (2013) "El descenso en las tasas de crecimiento demográfico y el menor ritmo de crecimiento de la productividad de los trabajadores hacen del ajuste al sistema de pensiones una tarea urgente en la agenda de los gobiernos".
Vale la pena resaltar que la presente investigación tiene como objetivo establecer una mesa de debate acerca del tema de la futura reforma pensional en Colombia, teniendo en cuenta la situación del sistema pensional, del mercado laboral y demográfica para lo primeros trece años del siglo XX. En esta línea, es pertinente mencionar que el país entra en un nuevo ciclo de recolección de datos en investigaciones como censo poblacional y la encuesta de presupuestos familiares, que afectarían notablemente los resultados del presente estudio. De acuerdo a lo anterior, es importante aludir que esta investigación continuara desarrollando nuevos documentos con base en los nuevos resultados estadísticos de las fuentes primarias.

\section{REFERENCIAS BIBLIOGRÁFICAS}

Acto Legislativo 01 de 2005, Diario Oficial de la República de Colombia, Bogotá D.C., 25 de Julio de 2005

ACUÑA, Diego, GONZÁLEZ, José, Luis, MONTOYA, Ana, María, PALACIO, Esteban \&REYES, Juan, David, (2009), "Salud y pensiones: los grandes retos de la política de seguridad social", 1ra Edición, Revista Supuestos, Universidad de los Andes

ALONSO, Meseguer, Javier \& HERCE, José, A, (2003), "Balance del sistema de pensiones y boom migratorio en España Proyecciones del modelo MODPENS"

ANTOLIN, P. (2008), "Ageing and the payout phase of pensions, annuities and financial markets", OECD Working Papers on Insurance and Private Pensions, No. 29, OECD publishing, (C) OECD. doi:10.1787/228645045336

ARANGO, Luis, Eduardo \& HAMANN, Franz, (2012) "El Mercado Laboral en Colombia Hechos, Tendencias e Instituciones”, Banco de la República, ISBN 987-958-664-261-3

ARANGO, Luis, Eduardo, ESCOBAR, Diana, Carolina, MONSALVE, Emma, Mercedes, (2013), "Subempleo por ingresos y funcionamiento del mercado de trabajo en Colombia", Borradores de Economía, No 764, Banco de la República.

ARIAS, Mauricio \& MENDOZA, Juan, Carlos, (2009), “Un modelo de simulación del régimen de ahorro individual con solidaridad en Colombia”, Reporte de Estabilidad Financiera, Banco de la República.

AYALA, Ulpiano \& ACOSTA, Olga, Lucía, (2002), "Políticas para promover una ampliación de la cobertura del sistema de pensiones en Colombia", Serie Financiamiento y Desarrollo No 118, Unidad de Estudios Especiales, Secretaria Ejecutiva, CEPAL 
BALAKRISHNAN, Pulapre, (2004), "Measuring Productivity in Manufacturing Sector", Economic and Political Weekly, Vol. 39, No. 14/15, pp. 1465-1471, http://www.jstor.org/stable/4414861

BERNAL, S., Raquel, (2009), "The Informal Labor Market in Colombia: identification and characterization", Desarrollo y Sociedad, 145, PP. 145-208, ISSN 0120-3584

BOLDRIN Michele, DOLADO, Juan, José; JIMENO, Juan, Francisco; \& PERACCHI Franco, (1999), “The Future of Pensions in Europe", Universidad Carlos III de Madrid and CEPR, Universidad Carlos III de Madrid and CEPR, Universidad Alcalá de Henares and CEPR, Universitá di Roma Tor Vergata1

BÖRSCH-Supan, Axel \& LUDWIG, Alexander, (2009), "Aging, Asset Markets, and Asset Returns: A View from Europe to Asia", Mannheim Research Institute for the Economics of Aging (MEA), University of Mannheim, Germany, National Bureau of Economic Research (NBER), Cambridge, Massachusetts

BUSTAMANTE, Izquierdo, Paola, (2006), "Factores que inciden en la cobertura del sistema pensional en Colombia”, Archivos de Economía, Documento 312,

CÁRDENAS Mauricio \& BERNAL Raquel, (2004), "Determinants of Labor Demand in Colombia 1976-1996", Chapter in NBER book Law and Employment: Lessons from Latin America and the Caribbean.

CEPAL (2009), "Envejecimiento, derechos humanos y políticas públicas", Publicación Naciones Unidas, ISBN: 978-92-1-323215-6

CESPEDES, Rangel, Erick, GONZALEZ, Quintero, Nestor, HERNÁNDEZ, Diaz, Gustavo, PIRAQUIVE, Galeano, Gabriel, RAMOS, Piracoca, Johanna, (2011), "Una mirada a la economía informal”, Documento 383, Archivos de Economía, Dirección de Estudios Económicos, Departamento Nacional de Planeación

CLAVIJO, Sergio, (1998), "Fiscal effect of the 1993 Colombian pension reform", Working Paper No 158, International Monetary Fund.

CLAVIJO, Sergio, (2009), "Social Security Reforms in Colombia: Striking Demographic and Fiscal Balances”, Working Paper No 58, International Monetary Fund.

CLAVIJO, Sergio, VERA, Alejandro, FANDIÑO, Alejandro, (2012), "La Desindustrialización en Colombia, Análisis Cuantitativo de sus Determinantes", Asociación Nacional de Instituciones Financieras-ANIF, ISBN 978-958-57042-2-0

CLAVIJO, Sergio, VERA, Alejandro, FANDIÑO, Alejandro, (2013), "Estructura Fiscal de Colombia y Ajustes Requeridos", Asociación Nacional de Instituciones Financieras-ANIF, ISBN 978-95857042-3-7

CONDE-Ruiz, J. Ignacio \& ALONSO, Javier, (2006), "El Sistema de Pensiones en España ante el reto del Envejecimiento", Fundación de Estudios de Economía Aplicada.

DÍAZ, Julio, López \& JIMÉNEZ, Ridruejo, Zenón (2001), "Pensiones crecimiento económico y envejecimiento poblacional", Departamento de Fundamentos del Análisis Económico, Universidad de Valladolid.

DOMEIJ, David \& FLODEN, Martin, (2003), "Population Aging and International Capital Flows", Stockholm School of Economics, Stockholm School of Economics and CEPR SSE/EFI Working Paper Series in Economics and Finance No 539 
ECHEVERRY, Garzón, Juan, Carlos, ESCOBAR, Arango, Andrés, MERCHAN, Hernández, César, PIRAQUIVE, Galeano, Gabriel, SANTA MARIA, Salamanca, Mauricio, (2001), "Elementos para el debate sobre una nueva reforma pensional en Colombia", Documento 156, Archivos de Economía, Dirección de Estudios Económicos, Departamento Nacional de Planeación

ECHEVERRY, Juan Carlos \& SANTAMARÍA, Mauricio, (2004), "The Political Economy of Labor Reform in Colombia", Documento CEDE 2004-22, ISSN 1657-7191

FLÓREZ, Carmen, Elisa (2000), "Las Transformaciones Sociodemográficas en Colombia durante el siglo XX”, Banco de la República, Tercer Mundo Editores, ISBN 958-601-872-5

FLÓREZ, Carmen, Elisa, (2002), "The function of the urban informal sector in employment. Evidence from Colombia 1984-2000", Documento CEDE 2002-04CEDE, Universidad de Los Andes, ISSN 1657-7191 (Edición electrónica)

FOUGÈRE, Maxime, MÉRETTE, Marcel (1999), "An Econometric Examination of the Impact of Population Ageing on Personal Savings in Canada", Department of Finance Working Paper 99-03.

HELMSDORFF, Loredana, (2007), "Ampliación de cobertura del Sistema Pensional colombiano y atención al adulto mayor", Estrategia para la Reducción de la Pobreza y la Desigualdad, Departamento Nacional de Planeación, ISBN: 978-958-8025-97-1

HIGGINS, Matthew \& WILLIAMSON, Jeffrey G., (1996), "Asian Demography and Foreign Capital Dependence", National Bureau of Economic Research, Working Paper No 5560

HIGGINS, Matthew. (1998), "Demography, national savings, and international capital flows", International Economic Review, 39(2), 343-369

ITO, Hiroyuki \& TABATA, Ken; (2010), "The spillover effects of population aging, international capital flows, and welfare", Journal of Population Economics, Volume 23, Issue 2, pp 665-702, 23:665-702 DOI 10.1007/s00148-009-0259-2

JIMENEZ-Ridruejo, Ayuso, Zenón, (2007),'La Sostenibilidad del Sistema de Pensiones en España: Envejecimiento, Inmigración Y Productividad", Universidad de Valladolid, Secretaria de Estado de la Seguridad Social, Dirección General de la Ordenación de la Seguridad Social, Ministerio de Trabajo y Asuntos Sociales.

JIMENO, Juan, Francisco., (2002), "Demografía, empleo, salarios y pensiones", Documento de Trabajo 2002-04, Fundación de Estudios de Economía Aplicada.

KATHURIA, Vinish, RAJ, Rajesh, S N \& SEN, Kunal, (2011), "Productivity measurement in Indian manufacturing: a comparison of alternative methods", Institute for Development Policy and Management (IDPM), Development Economics and Public Policy, Working Paper Series, The University of Manchester

LASSO, Valderrama, Francisco \& LÓPEZ, Castaño, Hugo, (2012), "El Mercado Laboral y el Problema Pensional Colombiano", Borradores de Economía \# 736, Banco de la República, Bogotá, Colombia.

Ley $N^{\circ} 789$, Diario Oficial de la República de Colombia, Bogotá D.C., 27 de Diciembre de 2007

Ley $N^{\circ}$ 797, Diario Oficial de la República de Colombia, Bogotá D.C., 29 de Enero de 2003

Ley N 860, Diario Oficial de la República de Colombia, Bogotá D.C., 29 de Diciembre de 2003 
LOAYZA, Norman, FAJNZYLBER, Pablo \& CALDERÓN, César, (2004), "Economic growth in Latin America and the Caribbean: stylized facts, explanations, and forecasts", Working Papers $\mathrm{N}^{\circ} 265$, Central Bank of Chile

LÓPEZ, Castaño, Hugo, (2012),"El mercado laboral colombiano: tendencias de largo plazo", En ARANGO, Luis, Eduardo \& HAMANN, Franz, "El Mercado Laboral en Colombia Hechos, Tendencias e Instituciones", Banco de la República, ISBN 987-958-664-261-3

LORA, Eduardo (2008), “Técnicas de Medición Económica, Metodología y Aplicaciones en Colombia”, Cuarte Edición, Editorial: Alfa Omega, ISBN 978-958-682-745-4

MINISTERIO DE TRABAJO (2006), "Resolución Numero 4316”, Bogotá, Colombia

MINISTERIO DEL TRABAJO (2013), “Nuevo modelo de protección para la vejez”, presentación oficial, Bogotá, 52 Diapositivas.

MORENO, Álvaro, Martín, \& ORTIZ, Fabio (2010), “Economía política de la reforma del sistema colombiano de pensiones”, Revista de Economía Institucional, vol.12, No 22, pp. 167-192

NACIONES UNIDAS, UN Population Information Network (POPIN), World Population Prospects: The 2012 Revision

NARCISO, Alexandre, (2010), "The impact of population ageing on international capital flows", Copenhagen Business School, MPRA Paper No. 26457, posted 8, http://mpra.ub.uni-muenchen. $\mathrm{de} / 26457 /$

OECD, (2011), "The economy of the possible: pensions and informality in Latin America", Working Paper No 295, Latin American Economic Outlook, OECD Development Centre.

OECD, (2015), "OECD Economic Surveys Colombia January 2015 overview”, OECD Economic Surveys, OECD Development Centre.

PARRA, Osorio, Juan, Carlos, (2001), "DNPENSION: Un modelo de simulación para estimar el costo fiscal del sistema pensional colombiano", Documento 150, Archivos de Economía, Dirección de Estudios Económicos, Departamento Nacional de Planeación

POTERBA, James, M. (2001), "Demographic structure and assets returns", Review of Economics and Statistics, 83(4), 565-584.

RADA, Codrina, (2009), "Introducing Demographic Changes in a Model of Economic Growth and Income Distribution", Working Paper No: 2009-01, Working Paper Series, Department of Economics, University of Utah

REYES, Sierra, Adriana Rocío, (2010), "Una aproximación al costo fiscal en pensiones como consecuencia del envejecimiento de la población en Colombia y el efecto de la sobre-mortalidad masculina", Facultad de Ciencias Económicas, Universidad Nacional de Colombia, Bogotá

RINCÓN, Hernán, RAMOS, Jorge, LOZANO, Ignacio, (2004), “Crisis Fiscal Actual: Diagnóstico y Recomendaciones", Borradores de Economía, No 145, Banco de la República.

RODRÍGUEZ, Oscar, ARÉVALO, Decsi, AYALA, Ulpiano, ARÉVALO, Edgar, Iván, (1992), "Estructura y Crisis de la Seguridad Social en Colombia (1946-1992)", Centro de Investigaciones para el Desarrollo, Facultad de Ciencias Económicas, Universidad Nacional de Colombia. ISBN 958-628-077-2 
ROMER, P. M. (1990),"Endogenous Technological Change”, Journal of Political Economy 98, S71-S102.

SALAZAR, Álvarez, Luz Day, (2007) "El envejecimiento en Colombia: Una Construcción Social 19702000", Tesis Doctoral, Departamento de Sociología y Análisis de las Organizaciones, Universidad de Barcelona

SÁNCHEZ, Torres, Roberto, Mauricio, (2008), "La crisis del sistema pensional colombiano. Análisis de cobertura y críticas a las fallidas reformas", Ensayos de Economía, No 32, 135·164, Universidad Nacional de Colombia.

SANDOVAL, Juan, Pablo, (2004) “Estructura Productiva, Mercado Laboral y Educación”, Working Paper, Facultad de Economía, Universidad Nacional de Colombia.

SANTA MARÍA, Mauricio, STEINER, Roberto, BOTERO Jorge Humberto, MARTÍNEZ Mariana, MILLÁN Natalia (2010), "El Sistema Pensional en Colombia: Retos y Alternativas para Aumentar la Cobertura, parte I, ¿cómo extender la cobertura de los programas de Capitalización individual?”, Fedesarrollo, Bogotá, Colombia

SANTA MARIA, Salamanca, Mauricio \& PIRAQUIVE, Galeano, Gabriel, (2013), "Evolución y alternativas del sistema pensional en Colombia”, Departamento de Planeación Nacional, Dirección de Estudios Económicos, Archivos de Economía, Documento 398.

SARGENT, Timothy, C. \& RODRIGUEZ, Edgard, R. (2001), "Labour or Total Factor Productivity: Do We Need to Choose?", Department of Finance Working Paper, Economic Studies and Policy Analysis Division, Economic and Fiscal Policy Branch, Department of Finance.

SCHMIDT, Hebbel, Klaus, (1997), "Fiscal and macroeconomic effects of Colombia's pension reforms 1997", Revista de Análisis Económico, Vol. 12, No 2, pp 37-66

SCHUTT, Pardo, Erika, (2011), "El salario mínimo y el régimen de ahorro individual en pensiones: diagnóstico, evidencia y problemática”, Documentos CEDE No 16, Universidad de Los Andes, ISSN 1657-7191

SUPAN, Axel Boersch-, LUDWIG, Alexander \& WINTER, Joachim, (2001), "Aging and International Capital Flows", NBER working paper series, National Bureau of Economic Research, Working Paper 8553, http://www.nber.org/papers/w8553

TORRES, Minoldo, María, Sol, \& PELÁEZ, Enrique, (2012), "El envejecimiento demográfico, ¿final de la seguridad social? Análisis en argentina y Latinoamérica”, CIECS-CONICET, Revista de ciencias sociales, ISSN 1696-7348

TURNER, Dave, GIORNO, Claude, DE SERRES, Alain, VOURC'H, Ann \& RICHARDSON, Pete, (1998), "The Macroeconomic Implications of Ageing in a Global Context", Economics Department Working Papers No. 193, OECD, ECO/WKP(98)6

URDINOLA, Piedad, (2011), "Colombia en el marco de: "Implications of the Financial Crisis for Long Run Retirement Security" O. Mitchell BY B., Presentación, 24 Diapositivas, Departamento de Estadíticas, Facultad de Ciencias, Universidad Nacional de Colombia.

URIBE. Mallarino. Consuelo, (2002), "La reforma de pensiones en Colombia y la equidad de género", Unidad Mujer y Desarrollo, Proyecto: Impacto de género de la reforma de pensiones en América Latina, CEPAL, ISBN 1680-8967. 
VIAL, Joaquín (2013), "Impactos económicos del envejecimiento de la población", Presentación, 32 Diapositivas, Inauguración año académico Facultad de Economía y Negocios, Universidad San Sebastián, Banco Central de Chile.

\section{Para citaciones:}

Perez-Vasquez, A., \& Sandoval-Castaño, J. (2017). Impactos del envejecimiento poblacional y de la dinámica del mercado laboral en el gasto público en pensiones en Colombia. Panorama Económico, 25, 4, pp. 493-518.

\section{AUTORES}

Andrés Pérez-Vasquez

Economista de la Universidad Santo Tomás (Colombia). Beneficiario de la Beca-Pasantía delPrograma Jóvenes Investigadores e Innovadores de la Convocatoria de Colciencias No. 566 de 2012.

Juan Sandoval-Castaño

Docente de la Universidad Nacional de Colombia. Magister en Economía de la Universidad de Amiens (Francia). Investigador del Grupo de Investigación en Socioeconomía, Instituciones y Desarrollo del Centro de Investigaciones para el Desarrollo de la Universidad Nacional de Colombia. 